\title{
Side-Chain Pruning Has Limited Impact on Substrate Preference in a Promiscuous Enzyme
}

\author{
Maximilian J. L. J. Fürst, ${ }^{\dagger}$ Elvira Romero, ${ }^{\dagger}$ J. Rúben Gómez Castellanos, $^{\ddagger}$ Marco W. Fraaije, ${ }^{*}{ }^{\dagger}$ \\ and Andrea Mattevi* $*$ \\ ${ }^{\dagger}$ Molecular Enzymology Group, University of Groningen, Nijenborgh 4, 9747AG, Groningen, The Netherlands \\ ${ }^{\ddagger}$ Department of Biology and Biotechnology, University of Pavia, Via Ferrata 1, 27100, Pavia, Italy
}

\section{Supporting Information}

ABSTRACT: Detoxifying enzymes such as flavin-containing monooxygenases deal with a huge array of highly diverse xenobiotics and toxic compounds. In addition to being of high physiological relevance, these drug-metabolizing enzymes are useful catalysts for synthetic chemistry. Despite the wealth of studies, the molecular basis of their relaxed substrate selectivity remains an open question. Here, we addressed this issue by applying a cumulative alanine mutagenesis approach to cyclohexanone monooxygenase from Thermocrispum municipale, a flavin-dependent Baeyer-Villiger monooxygenase which we chose as a model system because of its pronounced thermostability and substrate promiscuity. Simultaneous removal of up to eight noncatalytic active-site side chains including four phenylalanines had no effect on protein folding, thermo-

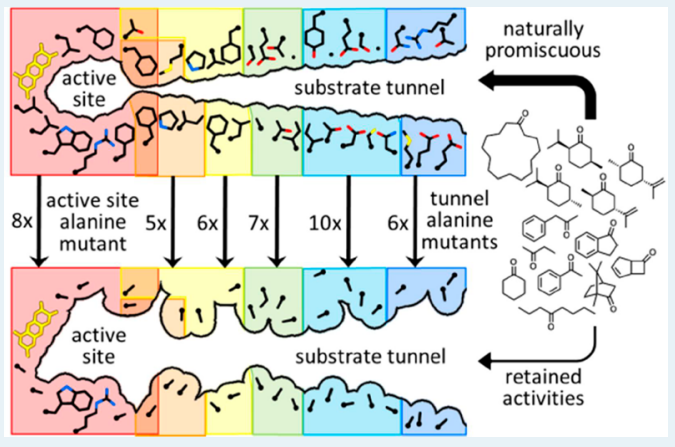
stability, and cofactor loading. We observed a linear decrease in activity, rather than a selectivity switch, and attributed this to a less efficient catalytic environment in the enlarged active-site space. Time-resolved kinetic studies confirmed this interpretation. We also determined the crystal structure of the enzyme in complex with a mimic of the reaction intermediate that shows an unaltered overall protein conformation. These findings led us to propose that this cyclohexanone monooxygenase may lack a distinct substrate selection mechanism altogether. We speculate that the main or exclusive function of the protein shell in promiscuous enzymes might be the stabilization and accessibility of their very reactive catalytic intermediates.

KEYWORDS: enzyme promiscuity, substrate specificity, active site, tunnel mutagenesis, alanine scanning mutagenesis, Baeyer-Villiger

\section{INTRODUCTION}

Enzymes are traditionally thought of as being very specific for the particular metabolic reaction they catalyze, and molecular discrimination between closely related compounds indeed often is an asset. However, it is also known that some degree of substrate promiscuity is common for most if not all enzymes, and this is assumed to be a prerequisite for their evolution. ${ }^{1}$ There are also enzymes equipped with an extremely broad specificity, and some of them have become trailblazers in modern biocatalysis. ${ }^{2}$ In many cases, the underlying physiological role can explain the selective advantage that led to the evolution of such indiscriminate catalysts. A classic example is detoxification, a process that involves diverse enzyme classes. ${ }^{3}$ In higher organisms, the metabolism of drugs proceeds via an initial modification phase involving cytochrome P450 (P450s) and flavin-containing monooxygenases (FMOs), while transferases and ligases flag a compound for decomposition or secretion in the subsequent conjugation phase. ${ }^{4}$ Performing these steps on a range of xenobiotics allows these enzymes to afford a broad protection. P450s were originally investigated because of their clinical relevance, ${ }^{5}$ but they also gained strong interest in biocatalysis and protein engineering, due to their unique catalytic competences. ${ }^{6}$ FMOs typically oxidize heteroatoms, ${ }^{7}$ but a human isoenzyme (FMO5) recently was shown to act as a Baeyer-Villiger monooxygenase (BVMO), ${ }^{8}$ adding to the repertoire of the reactions catalyzed by human detoxifying and drug/xenobiotic-metabolizing enzymes. BVMOs are enzymes present in all kingdoms ${ }^{9}$ and catalyze the incorporation of oxygen adjacent to a carbonyl moiety via their catalytic C4a-peroxyflavin intermediate, which results from the reaction of dioxygen $\left(\mathrm{O}_{2}\right)$ and reduced flavin adenine dinucleotide (FAD; Scheme 1). ${ }^{10}$

Interestingly, many characterized microbial BVMOs also display relaxed substrate specificities, even though they are typically thought to have a more specialized physiological role. Cyclohexanone monooxygenase (CHMO) from Acinetobacter sp. NCIMB 9871, for example, is part of a cyclohexanol degradation pathway, ${ }^{11}$ yet this enzyme was found to convert hundreds of different compounds. ${ }^{12,13}$ With several crystal structures $^{14-18}$ and a wealth of mutagenesis data at hand, ${ }^{19-24}$ BVMOs present an excellent model for the study of substrate acceptance and promiscuity. Engineering studies aiming at the

Received: September 20, 2018

Revised: October 26, 2018

Published: October 30, 2018 
Scheme 1. Baeyer-Villiger Reaction and Catalytic Mechanism of BVMOs Showing the Various Oxidation States of the Flavin (Fl)

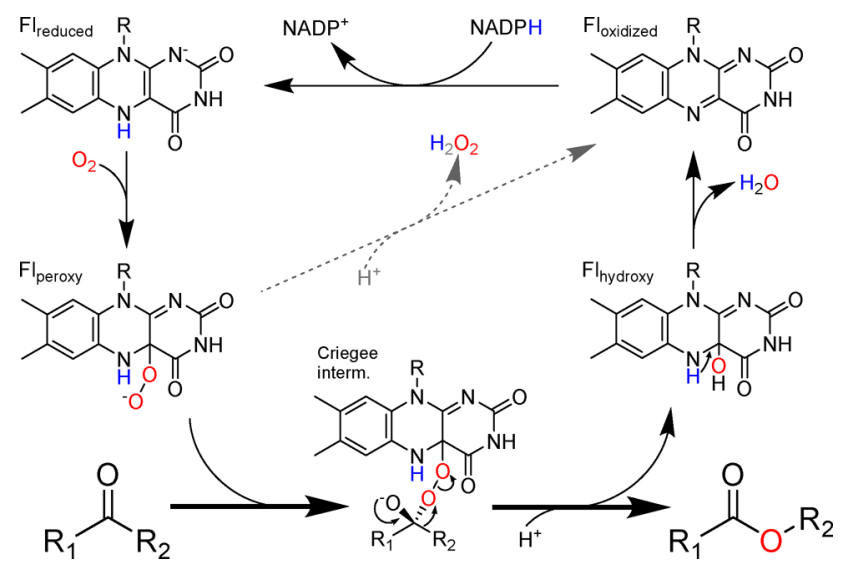

implementation of a BVMO for cyclohexanone conversion are particularly interesting; the resulting product, $\varepsilon$-caprolactone, is a precursor for nylon- 6 , a polymer produced industrially on a megaton scale. ${ }^{25}$

Several engineering attempts performed on a thermostable BVMO which is inactive on cyclohexanone (phenylacetone monooxygenase from Thermobifida $\mathrm{fusca}^{26}$ ) failed to generate a promiscuous mutant capable of acting on this substrate. ${ }^{21-23}$ The only mutant of phenylacetone monooxygenase known to convert small amounts of cyclohexanone combined mutations in the active site with mutations discovered through an unusual approach of induced allostery. ${ }^{24,27}$ The allosteric residues are approximately $18 \AA$ from the substrate binding site and may lead to a domain shift resulting in the alteration of the activesite shape. ${ }^{24}$ Conformational flexibility can indeed contribute to enzyme promiscuity ${ }^{28}$ and could also play a role in the kinetic mechanism of $\mathrm{BVMOs}^{18}$ as evidenced by crystal structures of CHMO, which show a rotation of the $\mathrm{NADP}^{+}$ domain of around $3^{\circ} .{ }^{14}$ The related proteins lysine monooxygenase and thioredoxin reductase show the same domain architecture and were found to rotate by $30^{\circ}$ and $67^{\circ}$, respectively. ${ }^{29,30}$ Although there is no structural evidence for similar movements in BVMOs, the inefficiency of rationally engineering phenylacetone monooxygenase might result from an incomplete picture of BVMO catalysis. Major shifts of the cofactor, ${ }^{14}$ complex loop rearrangements, ${ }^{31}$ and the lack of an explanation for the remote position of the conserved BVMO fingerprint motive ${ }^{18}$ leave room for speculations.

We therefore aimed to find evidence for so far unknown mechanisms involved in substrate preference and selectivity of BVMOs and chose a recently described thermostable CHMO as the target for this study. ${ }^{32}$ Because a lot of experimental data on single mutants of various CHMOs as well as phenylacetone monooxygenase can already be found in the literature (recently reviewed in ref 33), we undertook the uncommon approach of creating cumulative alanine mutants. Alanine lacks a distinct side chain and does not allow the backbone geometries of glycine. Thus, observed effects can largely be attributed to the loss of the wild-type side chain. ${ }^{34}$ In these minimalistic mutants, we explored the effects of incrementally removing those groups that shape the binding site and presumably establish favorable interactions with the substrates. While it was previously observed that enzymes possess backups and can compensate for the loss of some side chains through other residues or conformations, ${ }^{28}$ accumulating mutations quickly lead to inactivation. ${ }^{35}$ It was therefore against our expectations to observe that our radical side-chain pruning only had a strong effect on enzyme activity when applied to a massive extent. In combination with conclusions drawn from structural investigations, we show that in promiscuous enzymes such as BVMOs, the accessibility to enzyme-stabilized, yet chemically very reactive, flavin intermediates appears the main factor that governs substrate preference.

\section{RESULTS}

Structural Investigations Using a Substrate Mimic to Reveal Substrate Binding Modes. In BVMOs, the catalytic entity is the C4a-peroxyflavin, which reacts with the substrate to form the so-called Criegee intermediate (Scheme 1). ${ }^{10}$ Although the chemistry behind this reaction is well understood, crystal structures of monooxygenases with either flavin adduct are not available. It is possible that the unsuccessful protein engineering is a direct result of the uncertainty about the conformation of BVMOs in this critical catalytic step. Our first goal was, therefore, to rule out that BVMOs undergo a so far overlooked conformational change. Because both the C4aperoxyflavin as well as the Criegee intermediates seem too unstable to be crystallized and/or survive the X-ray exposure, we aimed to create a mimic of the C4a-peroxyflavin-substrate complex. In the course of our X-ray studies on a robust CHMO from Thermocrispum municipale (TmCHMO; Figure 1a), ${ }^{32}$ we noticed that an acetate molecule present in the

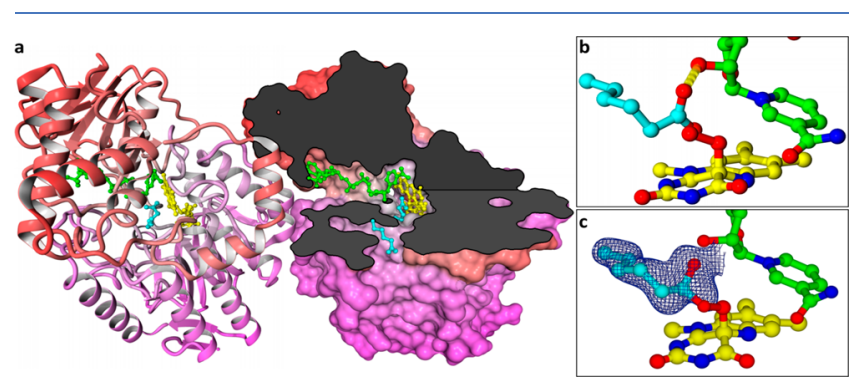

Figure 1. Overall structure of TmCHMO crystallized in complex with hexanoic acid (a, PDB code 6GQI) and superposition with a model of the peroxyflavin (b) and the Criegee intermediate (c). (a) Asymmetric unit of the TmCHMO crystal structure depicting the secondary structure (left monomer) and as surface representation (right monomer). The surface is cut open (gray planes) to emphasize the position of the ligand molecules in the tunnel and active site. The $\mathrm{NADPH}$ and FAD domains are colored red and pink, respectively. $\mathrm{NADP}^{+}, \mathrm{FAD}$, and hexanoic acids are shown as ball and sticks colored green, yellow, and cyan, respectively. (b and c) The flavin and $\mathrm{NADP}^{+}$ cofactors are shown as ball and sticks with yellow and green carbons, respectively. In b, hexanoic acid as crystallized is shown as ball and sticks (cyan carbons) superimposed on a model of the peroxyflavin. In c, a model of the Criegee intermediate (Scheme 1) of hexanal is shown together with the electron density of the bound hexanoic acid (weighted $2 F_{\mathrm{o}}-F_{\mathrm{c}}$ map, contoured at the $\sigma=1.2$ level; Table S1).

crystallization medium binds in front of the C4a atom of the flavin ring. As the Criegee intermediate is negatively charged and TmCHMO is active on linear aliphatic ketones, we reasoned that the carboxylate could mimic the intermediate. This idea prompted us to carry out cocrystallization trials with various carboxylic acids. The trials were successful with hexanoic acid, which bound to TmCHMO both in the tunnel leading toward the active site as well as in the active site itself 


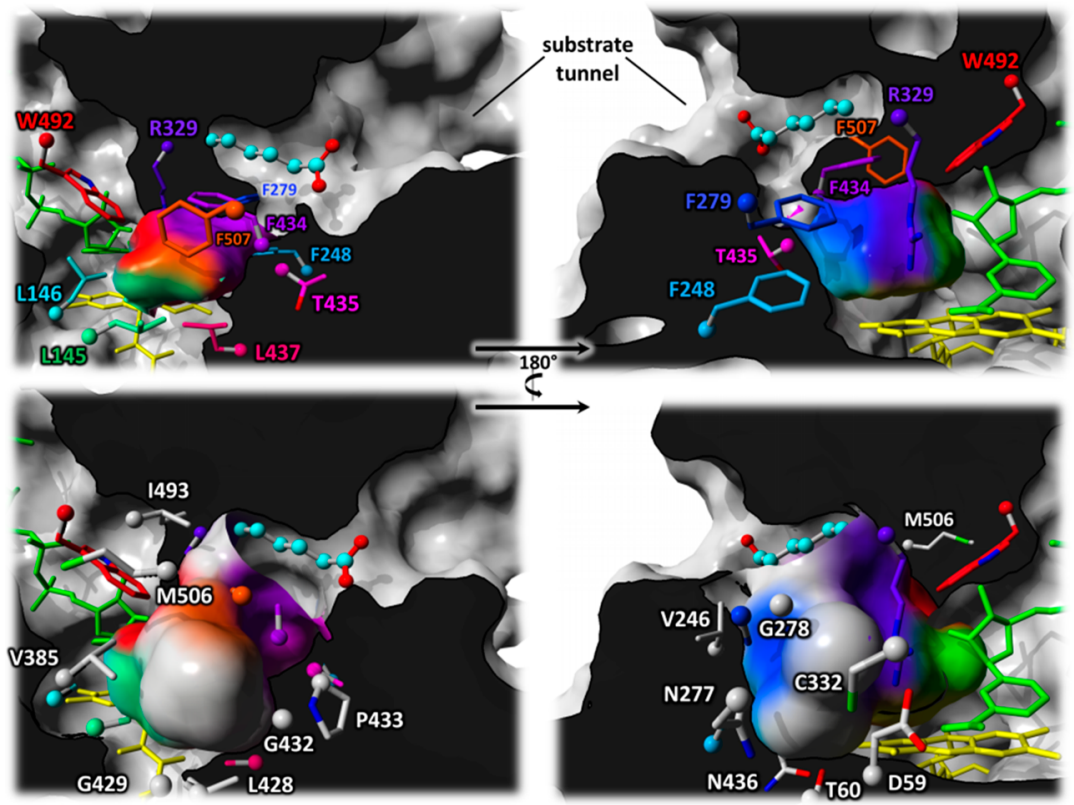

Figure 2. Active-site pocket and tunnel of TmCHMO wild type (crystal structure, top panel) and the $8 \times$ alanine mutant (model, bottom panel). The left and right panels are the same scene rotated by $180^{\circ}$. FAD and $\mathrm{NADP}^{+}$are depicted in yellow and green, respectively. All active-site residues are displayed as sticks in various colors. The surface they create and which forms the pocket is in the same color. In the mutant, residues that contribute to the newly shaped pocket are also shown with gray carbons. The hexanoic acid ligand bound in the tunnel is depicted as ball and sticks. The rest of the protein is shown as a gray surface representation, cut open at various planes (black). The active-site pocket is not cut, to emphasize the volume differences between wild type and mutant. As a result, the inner hexanoic acid ligand, bound close to the flavin (Figure $1 \mathrm{~b}, \mathrm{c}$ ), cannot be seen.

(Figure 1a; Table S1). The ligand close to the flavin bound in the same position as observed for previous BVMO crystal structure ligands (Figure S1) and in an orientation we would expect for the Criegee intermediate. For comparison, we computationally modeled the peroxyflavin and the Criegee intermediate (Figure $1 \mathrm{~b}, \mathrm{c}$ ). We found that the anionic peroxygroup stabilizes above the pyrimidine ring of the flavin, and this position coincides with one of the oxygen atoms of hexanoic acid in our structure (Figure 1b). The other carboxylate oxygen of hexanoic acid points away from the flavin to hydrogen bond with the hydrogens of the NE atom of Arg329 and of the $\mathrm{O} 1$ atom of $\mathrm{NADP}^{+}$. This position was previously predicted to be occupied by the negatively charged oxygen of the Criegee intermediate by a theoretical study ${ }^{36}$ (Figure 1c). Importantly, we found no conformational changes when comparing the overall protein arrangement of the TmCHMO-hexanoic acid complex with that of previously obtained structures. The $\mathrm{C} \alpha$-atom root-mean-square deviation from the previously obtained structure of TmCHMO in complex with $\mathrm{FAD}$ and $\mathrm{NADP}^{+}$in the oxidized (ligand-bound, PDB code 5M0Z) and reduced (different space group; 5M10) complexes was only $0.3 \AA$. We thus concluded that in the reaction outcome-determining catalytic step of the Criegee intermediate, the enzyme is unlikely to be in a different conformation than previously observed.

Pruning the Side Chains from the Substrate-Binding Site. Having ruled out large enzyme conformational changes during the Criegee intermediate step, we next turned our attention to the active-site pocket using the crystal structure as a reference. In TmCHMO, 10 residues form the catalytic site: Leu145, Leu146, Phe248, Phe279, Arg329, Phe434, Thr435, Leu437, Trp492, and Phe507 (Figure 2). Notably, these residues are strictly conserved in all bona fide CHMOs, i.e., closely related enzymes that were demonstrated to be active on cyclohexanone (Figure S2). Since it was shown that the strictly conserved aromatic residue at positions $492^{20}$ and $\operatorname{Arg} 329^{37}$ are essential for catalysis, these two positions were excluded for mutagenesis. The remaining eight residues were successively and cumulatively mutated to alanine. The order was chosen arbitrarily with the exception of the last added Leu146 and Leu437, which are within interacting distance from the two cofactors, and Thr435, whose side chain points away from the active site (Figure 2). Notably, four of the targeted residues are phenylalanines, resulting in a substantial increase in the volume of the active site upon mutation to alanine (Figure 2). Although the replacement of single active-site residues can lead to a more stable enzyme, ${ }^{38}$ previous studies showed that mutating the binding site residues in BVMOs usually slightly decreases enzyme stability. ${ }^{39}$ Moreover, accumulating mutations are frequently found to be deleterious in general. ${ }^{35}$ Therefore, we were expecting to encounter abolished activity at some point, but were hoping for retained protein folding ability.

We applied successive rounds of site-directed mutagenesis to a plasmid harboring the TmCHMO gene fused to a $\left(\mathrm{His}_{6}\right)$ SUMO expression tag. This resulted in eight variants ranging from the single $(1 \times)$ mutant L145A up to the 8-fold $(8 \times)$ mutant L145A/L146A/F248A/F279A/F434A/T435A/ L437A/F507A (Table S2). As they emerged in the course of mutagenesis, we also tested three more variants corresponding to alternative $2 \times, 5 \times$, and $7 \times$ mutants (Table 1 ). Upon transformation in Escherichia coli, we found that all variants expressed equally well as wild-type TmCHMO and could be purified loaded with $\mathrm{FAD}$. We determined the melting temperature $\left(T_{\mathrm{m}}\right)$ of the mutant proteins with a thermal shift assay that exploits flavin fluorescence (ThermoFAD). ${ }^{40}$ 
Table 1. Active-Site Residues Targeted in This Study (Grey Field Indicates a Mutation to Alanine in the Corresponding Variant)

\begin{tabular}{c|c|c|c|c|c|c|c|c|c|c|c|c}
\multicolumn{10}{c}{ Mutant } \\
Res
\end{tabular}

Despite the accumulating loss of active-site side chains, we found that the $T_{\mathrm{m}}$ gradually increased with each introduced alanine mutation until the $5 \times$ mutant, which had a $4{ }^{\circ} \mathrm{C}$ higher $T_{\mathrm{m}}$ than wild-type TmCHMO (Figure $3 \mathrm{a}$ ). Then, the stability slightly declined again until the $8 \times$ mutant, which still had a $3{ }^{\circ} \mathrm{C}$ higher $T_{\mathrm{m}}$ than the wild-type enzyme. From these data, we concluded that protein folding and stability were apparently not affected by the mutations.

We then wanted to determine the point of abolition of catalytic activity. To that end, we determined enzyme activity using a spectrophotometric NADPH consumption assay, but also performed bioconversions and product analysis via gas chromatography-mass spectrometry. Besides cyclohexanone, we also used rac-bicyclo-[3.2.0]hept-2-en-6-one, a model substrate for the analysis of stereo- and regioselectivity and readily converted by most BVMOs. Surprisingly, we found that none of the mutations caused a complete loss of activity. First, the kinetic analysis showed that the uncoupling rate, i.e., the unproductive NADPH oxidation, was barely affected by most mutations (Figure 3b, Table S2). Measurements in the presence of rac-bicyclo-[3.2.0]hept-2-en-6-one or cyclohexanone, on the other hand, showed an impaired catalytic performance: while the $1 \times$ mutant displayed a somewhat higher activity than the wild-type enzyme, the rates quickly declined upon accumulative loss of active-site side chains, until it became undistinguishable from the uncoupling rate from the $4 \times$ mutant on (Figure $3 b$ and Table S2). Likewise, the bioconversions showed the complete turnover of $1 \mathrm{mM}$ cyclohexanone in $24 \mathrm{~h}$ at $30{ }^{\circ} \mathrm{C}$ by all mutants up to and including the $4 \times$ mutant, yet also approximately $10 \%$ conversion by the $5 \times$ mutant, and trace conversions detectable up to the $8 \times$ mutant (Figure $3 \mathrm{c}$ ). Reactions with rac-bicyclo[3.2.0] hept-2-en-6-one were performed with $10 \mathrm{mM}$ substrate, and while the $4 \times$ mutant converted only traces, the two $5 \times$ (85\% and $29 \%)$ and the $6 \times$ mutant (97\%) still showed high turnovers (Figure $3 \mathrm{c}$ ). In the highest mutants, no product was detectable at first, but when we extended the reaction time to $72 \mathrm{~h}$ and supplemented the conversion mix with FAD and catalase, we could also detect traces of conversions of racbicyclo-[3.2.0]hept-2-en-6-one. These results led us to reject the hypothesis that a combination of side chains in the active site is required for catalysis in general, or for activity on
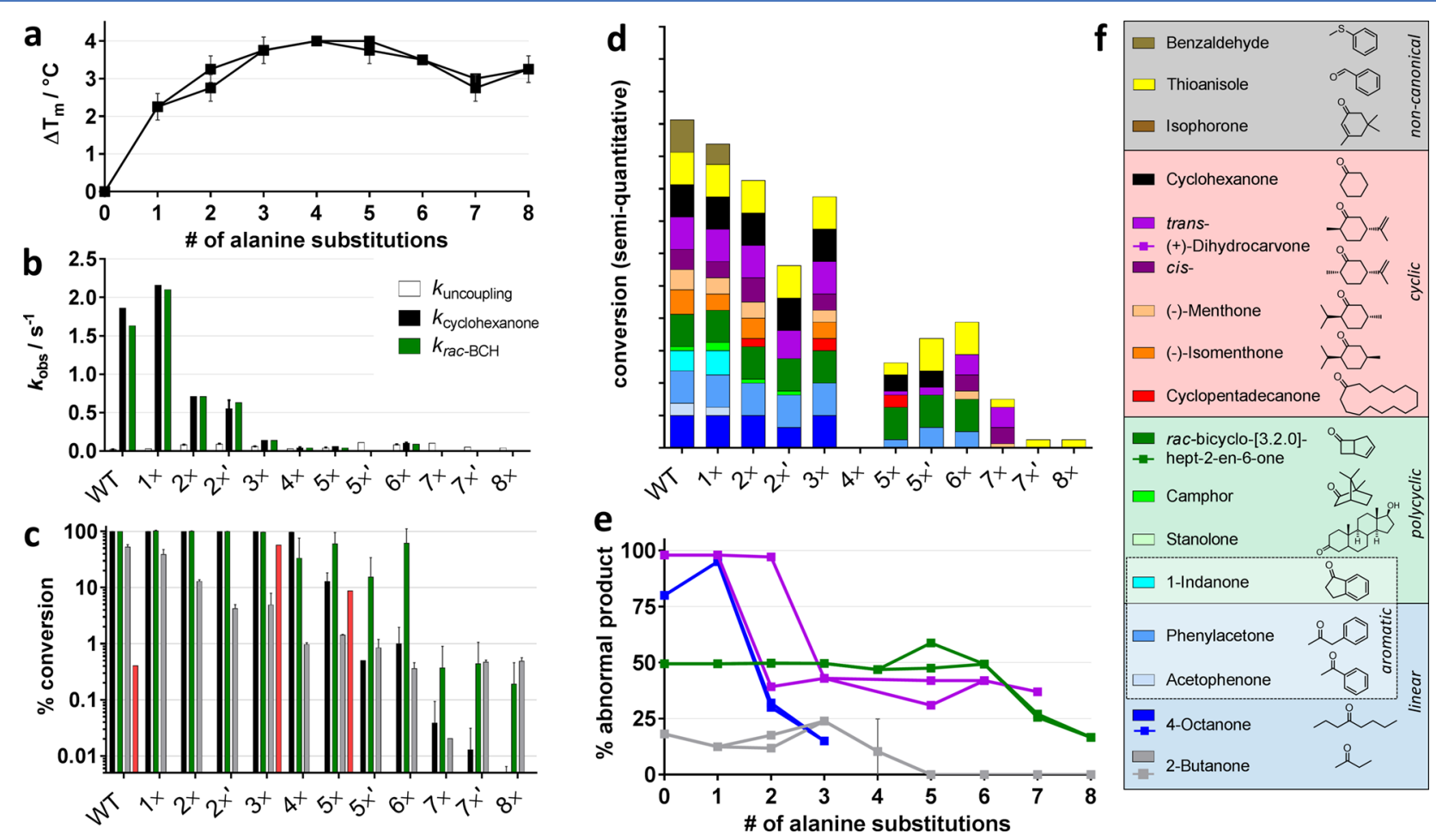

Figure 3. A summary of the thermostability, kinetic, and bioconversion properties of the TmCHMO active-site mutants (see Table 1). The thermostability and kinetic properties of the mutants are shown in a and b. BCH is rac-bicyclo-[3.2.0]hept-2-en-6-one. (c) Conversions of individual substrates are quantitative and plotted on a logarithmic scale. The enzyme concentration was $2 \mu \mathrm{M}$. 2-Butanone conversions were performed using whole cells. More information can be found in the Supporting Information. (d) Conversions of substrate mixes are semiquantitative and approximated based on GC peaks as full ( $>99 \%)$, moderate (50-99\%), low (5-50\%), or trace $(<5 \%)$. The enzyme concentration was $10 \mu \mathrm{M}$. Panel e illustrates product regioselectivity (abnormal vs normal lactone). (f) Legend for $\mathrm{c}-\mathrm{e}$. All data sets with an error bar (corresponding to $\pm \mathrm{sd}$ ) are from two, the remaining data from one independent experiment. 


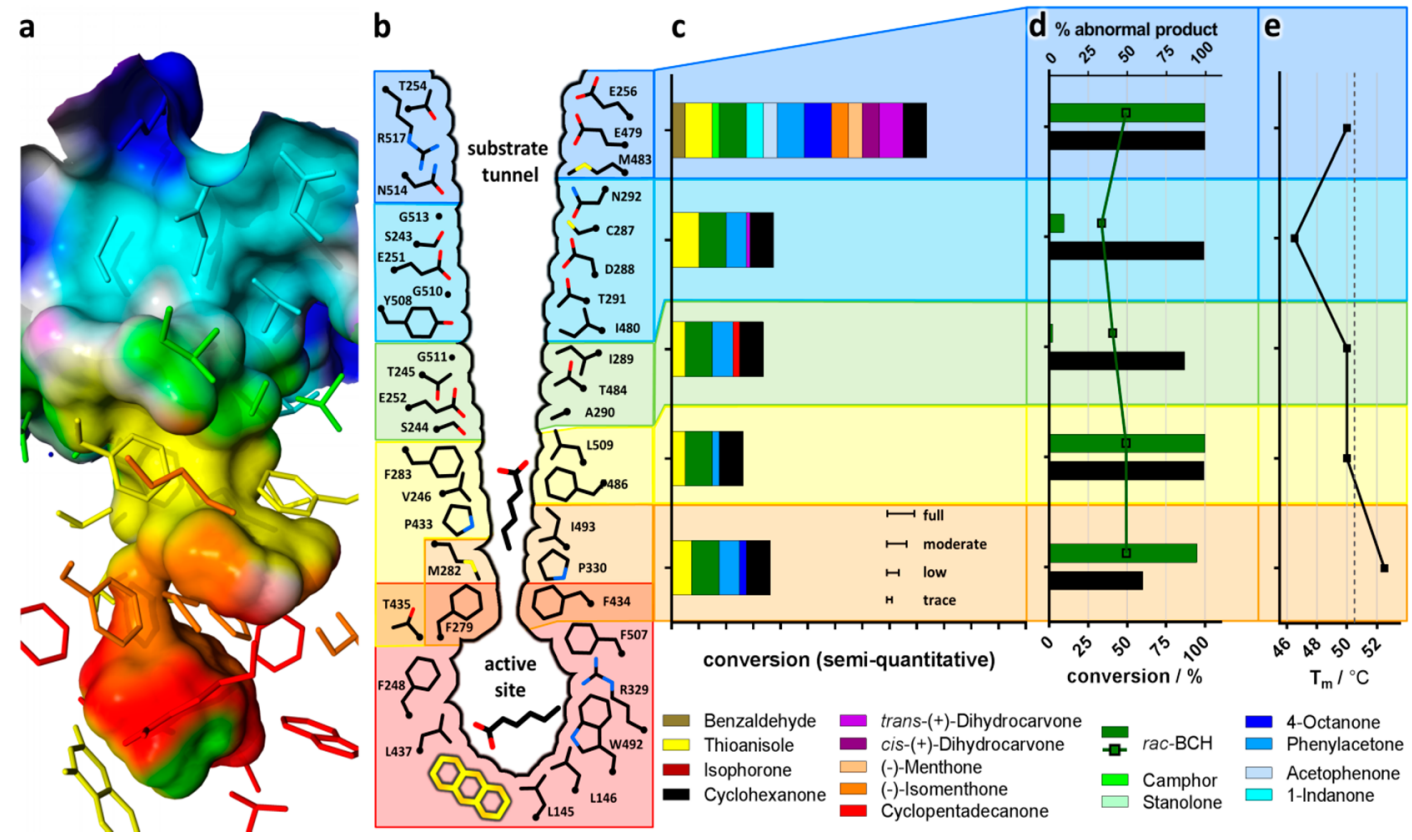

Figure 4. Tunnel mutagenesis strategy and activity results. (a) Surface representation of the substrate tunnel of TmCHMO occurring in the crystal structure. (b) Schematic cross-section through TmCHMO's active site and substrate tunnel and contributing residues. All residues depicted in the same color and corresponding to ring-like dissections of the tunnel were simultaneously mutated to alanine. (c and d) Conversion results for substrate mixes (c, semiquantitative, see Figure 3) and of cyclohexanone and rac-bicyclo-[3.2.0]hept-2-en-6-one (rac-BCH) in individual conversions (d, quantitative). Enzyme concentrations were $2 \mu \mathrm{M}$ and $10 \mu \mathrm{M}$ for mixed and individual substrates, respectively. (e) Stability of the ring-wise tunnel multialanine mutants; the dashed line indicates the melting temperature of the wild-type enzyme.

cyclohexanone in particular. In fact, these results indicate that none of the targeted residues is strictly essential for either task.

We also analyzed the effect on CHMO's substrate promiscuity and performed conversions with two substrate mixes. These contained common BVMO substrates [4octanone, phenylacetone, (+)-dihydrocarvone], as well as substrates for noncanonical oxidations (thioanisole, benzaldehyde, isophorone) together with compounds typically not accepted by CHMOs [(-)-menthone, (-)-isomenthone, acetophenone, 1-indanone, cyclopentadecanone, camphor, stanolone]. The results are summarized in Figure $3 \mathrm{~d}$. The substrate scope of wild-type TmCHMO was found to be even broader than assumed. Besides converting cyclic substituted and linear ketones and performing sulfoxidations, TmCHMO also oxidizes benzaldehyde and-in contrast to CHMO from Acinetobacter sp. NCIMB $9871^{41}$ - converts aromatic ketones such as 1-indanone and acetophenone. The only tested substrates that were not converted were very bulky or $\alpha, \beta$ unsaturated ketones. While previous work already showed a high affinity of wild-type TmCHMO for some of the assayed compounds, $^{32}$ the detection of a comparable amount of product resulting from the conversion of the newly identified substrates suggest that their binding constants are on the same order of magnitude. The alternative scenario, i.e., very high $k_{\text {cat }}$ and $K_{\mathrm{M}}$ values, is improbable taking into account that wild-type TmCHMO shows similar $k_{\text {cat }}$ values (and thus the same ratelimiting step) with most of the assayed substrates. In the mutants, on the other hand, the promiscuous activity gradually vanishes. Although sulfoxidation capability and activity on cyclic ketones is mostly retained in the highest mutants, activity on nearly all other substrates is practically lost. The $4 \times$ mutant showed no activity in these conversions; apparently this particular combination of mutations is unfavorable in some conditions (presumably in the presence of the more elevated concentration of acetonitrile used in these reactions). Notably, for some mutants we observed an activity on cyclopentadecanone, a transformation that was never previously reported for a CHMO. When testing these mutants in individual conversions with $1 \mathrm{mM}$ of this bulky substrate, the $3 \times$ mutant converted approximately $60 \%$ to the corresponding lactone (Figures 3c, S3).

Because some of the substrates we used can yield two different products depending on the side of oxygen incorporation, we could also study the effect of the mutations on regioselectivity. We found that if the wild-type enzyme produced exclusively the normal product (where the oxygen is incorporated next to the more substituted carbon), the regioselectivity remained unaffected in the mutants. When the enzyme produced fully or partially the abnormal product, however, the selectivity turned in favor of the normal product as more side chains were removed. As is the case for other CHMOs, ${ }^{42}$ wild-type TmCHMO produces exclusively the abnormal lactone from trans-(+)-dihydrocarvone. In the $1 \times$ and one of the $2 \times$ mutants, this selectivity is retained, while all the other mutants produce between 31 and $43 \%$ abnormal lactone. This apparent on/off mechanism seems to depend on Phe279: if it is present, regioselective production of the abnormal lactone occurs, while in its absence the residual production is unselective and approximates the value found for Baeyer-Villiger reactions via chemical oxidation. ${ }^{43}$ For 4octanone, the abnormal production slightly increases upon the first mutation approximating full regioselectivity but then drops to 32 and $15 \%$ in the $2 \times$ and $3 \times$ mutants. Using a whole cell assay, we also performed conversions with $11 \mathrm{mM}$ 2-butanone (Figure 3c), which yields the industrially relevant methyl propanoate when the abnormal product is formed. We found exclusively the normal product from the $5 \times$ mutant on (Figure 3e). Wild-type TmCHMO converts rac-bicyclo-[3.2.0] hept-2- 
en-6-one regiodivergently, leading to the abnormal lactone from one substrate enantiomer, and the normal lactone from the other. We found that the enzyme was able to retain this behavior until the $6 \times$ mutant. Previous protein engineering studies aiming to change the regioselectivity in BVMOs often sought to rationalize the switch toward normal lactone production by computational models. ${ }^{44-46}$ Our findings support the simple conclusions that, in a spacious and nonspecific binding site, the normal product is preferentially formed, leading to the same regioselectivity as the nonenzymatic Bayer-Villiger reaction. The shift toward the abnormal product can thereby take place only in the context of a sterically restrained environment. A recent study used extensive computational analysis to investigate the origin of abnormal product formation in TmCHMO mutants and corroborates this conclusion. ${ }^{47}$

Expanding to the Active-Site Tunnel. Our results indicate that the active-site residues are not strictly essential for catalysis and contribute to CHMO's substrate preference only to a limited extent since most mutants retained a broad substrate scope with highest activities toward cyclohexanone and thioanisole. If this selectivity was not determined in the active site, however, it consequently would have to be established elsewhere. A hint about this hypothesis was given by our crystal structure of TmCHMO in complex with hexanoic acid. The asymmetric unit of the crystals contains two enzyme monomers. Both monomers contain the hexanoic ligand in the active site, but one of them also features a defined density for the same ligand in the tunnel that connects the active site to the solvent (Figures 2, 4a). This tunnel has previously been observed in BVMOs, ${ }^{17,48}$ but our structure is a unique capture of a molecule along this diffusion pathway.

This newly emerged evidence for the importance of the tunnel prompted us to investigate its role in substrate acceptance. After a careful visual inspection, we defined as tunnel residues only those amino acids that protrude with their side chain into the path of the tunnel. Residues contributing with their backbone only were excluded, because mutagenesis would not affect the tunnel shape and easily disturb the hydrophobic packing. The beginning of the tunnel was defined as the rim at the solvent exposed outside and the end as the catalytic site (Figures 2 and 4).

If the tunnel indeed was partially or fully responsible for CHMO's activity on cyclohexanone, we hypothesized that we could also transfer this activity by transplanting the tunnel. As an engineering target, we chose phenylacetone monooxygenase, which is inactive on cyclohexanone and shares $44 \%$ sequence identity with TmCHMO. Because some active-site residues also partially contribute to the shape of the tunnel further up, we created two variants for the transplantation approach: one with those residues exchanged that only form the tunnel, and one in which also the entire catalytic site was exchanged (Tables S3-S5). The latter mutant included the double deletion of Ser441 and Ala442, the so-called active-site "bulge" present in phenylacetone monooxygenase, ${ }^{21}$ and two mutations corresponding to amino acids that only contribute to the active site found in phenylacetone monooxygenase (Ile339 and Leu340). Similarly, Phe250 does not contribute to the tunnel in TmCHMO, but because the corresponding Arg258 in phenylacetone monooxygenase does, it was also exchanged in both variants. The two enzymes furthermore display several glycine insertions and deletions in the tunnel and/or the active site, and those were also exchanged (details can be found in the Supporting Information). The resulting $25 \times$ and $38 \times$ mutant genes of phenylacetone monooxygenase were ordered as synthetic genes, cloned and transformed, and expressed in E. coli. We found expression and flavin incorporation unaffected, but when we tried the proteins in bioconversions, only the $25 \times$ mutant showed minor activity with rac-bicyclo-[3.2.0] hept-2-en-6-one, which was converted to the two normal lactones (Table S6). No activity was found with any of the other substrates. Clearly, simply transplanting the tunnel-surface residues from one enzyme to the other was not causing a transfer of substrate selectivity. On the other hand, it is remarkable that such an overwhelming set of mutations did not abolish FAD binding and protein stability (Table S6), highlighting a remarkable degree of structural tolerance featured by the BVMO protein scaffold.

As a second strategy, we followed our previous approach of cumulative alanine substitutions. We dissected the tunnel in five ring-like sections, because a mutation of all residues to alanine at once was likely to lead to insoluble protein. Each section contained 5-10 residues, and those were simultaneously mutated to alanine (Figure $4 a$ ). We created the plasmids using a PCR based method using mutated primers and subsequent Gibson assembly. ${ }^{49}$ The proteins expressed well with incorporated flavin. The more external ring-1 residues turned out to have little influence on the enzyme properties (Figure $4 b, c$ ). Conversely, the other mutants were mostly inactive on substituted cyclic, aromatic, and linear ketones. However, they retained a high activity on cyclohexanone and moderate activities on thioanisole, rac-bicyclo[3.2.0] hept-2-en-6-one, 4-octanone, and acetophenone (Figure $4 \mathrm{c}$ ). In the case of ring 3 , the mutant was unable to convert more than small amounts of rac-bicyclo-[3.2.0]hept-2-en-6-one (Figure 4c), but it gained weak activity on cyclopentadecanone (Figure $4 \mathrm{~b}$ ), possibly indicating that this particular part of the tunnel presents a bottleneck for the entry of bulky substrates. Low activity on rac-bicyclo-[3.2.0] hept-2-en-6-one was also found for the ring 2 multiple-Ala mutant, but this could result from its decreased stability (Figure $4 \mathrm{~d}$ ). The regioselectivity remained relatively unaffected in these mutants (Figure 4c). In general, these experiments followed the same trend observed for the active-site poly-Ala mutants. Introducing multiple alanine residues on the tunnel wall does not affect the preference for cyclohexanone, thioanisole, and rac-bicyclo[3.2.0] hept-2-en-6-one, which remain the best substrates for all mutants despite their small bulkiness.

Influence on the Flavin-Peroxide Stabilization. For a more in-depth understanding of the effect of side-chain pruning on the catalytic cycle, we used a stopped-flow spectrophotometer to study both the reductive and oxidative half-reactions of the $6 \times$ variant, which is the highest mutagenized enzyme variant with well detectable activity (Figure 3). The mutant became fully reduced in the presence of NADPH under anaerobic conditions, as evidenced by the loss of the absorbance peak at $440 \mathrm{~nm}$ (Figure S4). The stopped-flow traces at $440 \mathrm{~nm}$ were best fit to a double exponential function with an initial faster phase accounting for $71 \%$ of the total absorbance change. The corresponding $k_{\text {red } 1}$ and $k_{\text {red } 2}$ values were 0.2 and $0.02 \mathrm{~s}^{-1}$, respectively. These data can be compared with the stopped-flow traces at $440 \mathrm{~nm}$ for wild-type TmCHMO, which were best fit to a triple exponential function with $k_{\text {red }}$ values of 30,2 , and $0.1 \mathrm{~s}^{-1}$. The $K_{\mathrm{d}}$ value for NADPH was too small to be determined under pseudo-first-order conditions ( $\geq 5$-fold excess of 
NADPH over enzyme) for both the $6 \times$ and wild-type enzymes. To study the oxidative half-reaction, the $6 \times$ variant was anaerobically mixed with a stoichiometric amount of NADPH. Next, the resulting two-electron reduced enzyme was reacted with an air-saturated buffer resulting in an immediate increase in absorbance at $440 \mathrm{~nm}$ (Figure S4). The trace was best fit to a double exponential function corresponding to rates of 0.4 and $0.1 \mathrm{~s}^{-1}$. Differently from wild-type TmCHMO, ${ }^{32}$ a stable C4a-peroxyflavin with an absorption maximum at around 355 $\mathrm{nm}$ was not observed in the $6 \times$ variant at either 25 or $4{ }^{\circ} \mathrm{C}$, most likely because its decay is faster than its formation. Collectively, these experiments showed that both the reductive and the oxidative half-reactions are impaired in the $6 \times$ variant. This may be due to a nonoptimal position of the NADPH in the enzyme, which hampers both hydride transfer and stabilization of the C4a-peroxyflavin. Nevertheless, the mutants must retain some stabilization of the C4a-peroxyflavin (documented by the detection of Baeyer-Villiger reaction products in the above bioconversions; Figures 3 and 4), giving further evidence that the intermediate is above all stabilized by the bound $\mathrm{NADP}^{+}$, potentially supported by residues at the flavin's si side.

\section{DISCUSSION}

Our results from the mutagenesis of the active site and the tunnel collectively suggest that we were not targeting a structural entity that is fully responsible for the selectivity differences in BVMOs. Although similar approaches to our tunnel transplantation attempt have failed also in other cases, ${ }^{50}$ our section-wise "alanization" clearly shows the retention of activity with cyclohexanone and other good substrates. Although we observe decreased substrate promiscuity, these effects are likely to stem from an overall reduced catalytic efficiency, as evidenced by the reduced observed rates. Since the activity on cyclohexanone is not overwhelmingly determined by CHMO's active site or tunnel, it is possible that there is another, more remote substrate recognition and filter site. This could potentially also involve protein dynamics that previous BVMO structures were simply unable to capture. The second and-according to Occam's razor-more likely possibility is that CHMOs simply lack a selection mechanism entirely. Both BVMOs and flavin-containing monooxygenases were previously described as "cocked guns," their continuous stabilization of the C4a-peroxyflavin, which readily reacts with any substrate reaching the active site. Our experiments expand the idea to a "cocked shotgun" and suggest that enzymes like CHMO simply provide a scaffold to stabilize the reactive cofactor, and potentially lack a specific mechanism for substrate uptake and acceptance entirely. The only filter would then be the size of the active site and the increasing hydrophobicity toward the inside. Because most biological metabolites are polar, this rather crude selection of favoring partition from the solvent might be sufficient to prevent these enzymes from unwanted cellular reactions.

Promiscuous activity on polar compounds can easily interfere with primary metabolism; thus, enzymatic activity on such molecules needs to be more tightly regulated. On the one hand, enzymes can evolve specific mechanisms that limit broad specificity, as is the case for BVMO-related hydroxylases/monooxygenases. ${ }^{52}$ Accumulation of paralogs with a more restricted substrate scope can achieve diversity without complete unspecificity, as could for example be the case for a set of BVMOs found in a single plant biomass-degrading bacterial species. $^{41,53}$ On the other hand, expression of promiscuous isoforms can be restricted to certain tissues, ${ }^{54}$ or regulation can occur through wide substrate-dependent variations in the catalytic rate, as has been observed for detoxifying paraoxonases. ${ }^{55}$ An "excluding rather than binding" mechanism as we show is the case for some BVMOs, however, could well be the case for many catabolic or detoxifying enzymes which show high promiscuity toward hydrophobic substrates, such as flavin-containing monooxygenases ${ }^{56}$ or cytochrome P450s. ${ }^{57}$ Our completely "alanized" active-site mutant of TmCHMO can also be a useful starting point for engineering novel activities, since it can be regarded as a minimal active-site scaffold.

\section{ASSOCIATED CONTENT}

\section{Supporting Information}

The Supporting Information is available free of charge on the ACS Publications website at DOI: 10.1021/acscatal.8b03793.

Experimental procedures, tunnel exchange mutagenesis, supporting figures and tables (PDF)

\section{AUTHOR INFORMATION}

\section{Corresponding Authors}

*E-mail: m.w.fraaije@rug.nl.

*E-mail: andrea.mattevi@unipv.it.

ORCID

Marco W. Fraaije: 0000-0001-6346-5014

Andrea Mattevi: 0000-0002-9523-7128

\section{Author Contributions}

M.J.L.J.F., E.R., M.W.F., and A.M. wrote the manuscript. M.J.L.J.F and E.R. performed all experiments. J.R.G.G. conducted the X-ray crystallography studies.

\section{Funding}

The research for this work received funding from the European Union (EU) project ROBOX (grant agreement no. 635734) under EU's Horizon 2020 Programme Research and Innovation actions H2020-LEIT BIO-2014-1.

\section{Notes}

The authors declare no competing financial interest.

\section{ACKNOWLEDGMENTS}

We acknowledge the European Synchrotron Radiation Facility and the Swiss Light Source for the provision of beam time.

\section{REFERENCES}

(1) Khersonsky, O.; Tawfik, D. S. Enzyme Promiscuity: A Mechanistic and Evolutionary Perspective. Annu. Rev. Biochem. 2010, 79, 471-505.

(2) Turner, N. J. Directed Evolution Drives the Next Generation of Biocatalysts. Nat. Chem. Biol. 2009, 5, 567.

(3) Iyanagi, T. Molecular Mechanism of Phase I and Phase II DrugMetabolizing Enzymes: Implications for Detoxification. In International Review of Cytology; Academic Press: New York, 2007; pp 35112

(4) Xu, C.; Li, C. Y.; Kong, A. N. Induction of Phase I, II and III Drug Metabolism/Transport by Xenobiotics. Arch. Pharmacal Res. 2005, 28, 249-268.

(5) Guengerich, F. P. Human Cytochrome P450 Enzymes. In Cytochrome P450; Springer: Berlin, 2005; pp 377-530.

(6) Jung, S. T.; Lauchli, R.; Arnold, F. H. Cytochrome P450: Taming a Wild Type Enzyme. Curr. Opin. Biotechnol. 2011, 22, 809817. 
(7) Phillips, I. R.; Shephard, E. A. Drug Metabolism by FlavinContaining Monooxygenases of Human and Mouse. Expert Opin. Drug Metab. Toxicol. 2017, 13, 167-181.

(8) Fiorentini, F.; Geier, M.; Binda, C.; Winkler, M.; Faber, K.; Hall, M.; Mattevi, A. Biocatalytic Characterization of Human FMO5: Unearthing Baeyer-Villiger Reactions in Humans. ACS Chem. Biol. 2016, 11, 1039-1048.

(9) Mascotti, M. L.; Lapadula, W. J.; Juri Ayub, M. The Origin and Evolution of Baeyer-Villiger Monooxygenases (BVMOs): An Ancestral Family of Flavin Monooxygenases. PLoS One 2015, 10, e0132689.

(10) Sheng, D.; Ballou, D. P.; Massey, V. Mechanistic Studies of Cyclohexanone Monooxygenase: Chemical Properties of Intermediates Involved in Catalysis. Biochemistry 2001, 40, 11156-11167.

(11) Iwaki, H.; Hasegawa, Y.; Teraoka, M.; Tokuyama, T.; Bergeron, H.; Lau, P. C. Identification of a Transcriptional Activator (ChnR) and a 6-Oxohexanoate Dehydrogenase ( $\mathrm{ChnE}$ ) in the Cyclohexanol Catabolic Pathway in Acinetobacter Sp. Strain Ncimb 9871 and Localization of the Genes That Encode Them. Appl. Environ. Microbiol. 1999, 65, 5158-5162.

(12) Mihovilovic, M. D.; Müller, B.; Stanetty, P. MonooxygenaseMediated Baeyer-Villiger Oxidations. Eur. J. Org. Chem. 2002, 2002, $3711-3730$.

(13) Stewart, J. D. Cyclohexanone Monooxygenase: A Useful Reagent for Asymmetric Baeyer-Villiger Reactions. Curr. Org. Chem. 1998, 2, 195-216.

(14) Mirza, I. A.; Yachnin, B. J.; Wang, S.; Grosse, S.; Bergeron, H.; Imura, A.; Iwaki, H.; Hasegawa, Y.; Lau, P. C.; Berghuis, A. M. Crystal Structures of Cyclohexanone Monooxygenase Reveal Complex Domain Movements and a Sliding Cofactor. J. Am. Chem. Soc. 2009, 131, 8848-8854.

(15) Yachnin, B. J.; McEvoy, M. B.; MacCuish, R. J.; Morley, K. L.; Lau, P. C.; Berghuis, A. M. Lactone-Bound Structures of Cyclohexanone Monooxygenase Provide Insight into the Stereochemistry of Catalysis. ACS Chem. Biol. 2014, 9, 2843-2851.

(16) Yachnin, B. J.; Sprules, T.; McEvoy, M. B.; Lau, P. C.; Berghuis, A. M. The Substrate-Bound Crystal Structure of a Baeyer-Villiger Monooxygenase Exhibits a Criegee-Like Conformation. J. Am. Chem. Soc. 2012, 134, 7788-7795.

(17) Orru, R.; Dudek, H. M.; Martinoli, C.; Torres Pazmino, D. E.; Royant, A.; Weik, M.; Fraaije, M. W.; Mattevi, A. Snapshots of Enzymatic Baeyer-Villiger Catalysis: Oxygen Activation and Intermediate Stabilization. J. Biol. Chem. 2011, 286, 29284-29291.

(18) Malito, E.; Alfieri, A.; Fraaije, M. W.; Mattevi, A. Crystal Structure of a Baeyer-Villiger Monooxygenase. Proc. Natl. Acad. Sci. U. S. A. 2004, 101, 13157-13162.

(19) Torres Pazmiño, D. E.; Snajdrova, R.; Rial, D. V.; Mihovilovic, M. D.; Fraaije, M. W. Altering the Substrate Specificity and Enantioselectivity of Phenylacetone Monooxygenase by StructureInspired Enzyme Redesign. Adv. Synth. Catal. 2007, 349, 1361-1368.

(20) Dudek, H. M.; Fink, M. J.; Shivange, A. V.; Dennig, A.; Mihovilovic, M. D.; Schwaneberg, U.; Fraaije, M. W. Extending the Substrate Scope of a Baeyer-Villiger Monooxygenase by Multiple-Site Mutagenesis. Appl. Microbiol. Biotechnol. 2014, 98, 4009-4020.

(21) Bocola, M.; Schulz, F.; Leca, F.; Vogel, A.; Fraaije, M. W.; Reetz, M. T. Converting Phenylacetone Monooxygenase into Phenylcyclohexanone Monooxygenase by Rational Design: Towards Practical Baeyer-Villiger Monooxygenases. Adv. Synth. Catal. 2005, 347, 979-986.

(22) Reetz, M. T.; Wu, S. Greatly Reduced Amino Acid Alphabets in Directed Evolution: Making the Right Choice for Saturation Mutagenesis at Homologous Enzyme Positions. Chem. Commun. 2008, 0, 5499-5501.

(23) Reetz, M. T.; Wu, S. Laboratory Evolution of Robust and Enantioselective Baeyer-Villiger Monooxygenases for Asymmetric Catalysis. J. Am. Chem. Soc. 2009, 131, 15424-15432.

(24) Wu, S.; Acevedo, J. P.; Reetz, M. T. Induced Allostery in the Directed Evolution of an Enantioselective Baeyer-Villiger Monooxygenase. Proc. Natl. Acad. Sci. U. S. A. 2010, 107, 2775-2780.
(25) Arpe, H.-J. Industrial Organic Chemistry, 5th ed.; Wiley-VCH Verlag GmbH: Weinheim, Germany, 2010.

(26) Fraaije, M. W.; Wu, J.; Heuts, D. P.; van Hellemond, E. W.; Spelberg, J. H.; Janssen, D. B. Discovery of a Thermostable BaeyerVilliger Monooxygenase by Genome Mining. Appl. Microbiol. Biotechnol. 2005, 66, 393-400.

(27) Parra, L. P.; Acevedo, J. P.; Reetz, M. T. Directed Evolution of Phenylacetone Monooxygenase as an Active Catalyst for the BaeyerVilliger Conversion of Cyclohexanone to Caprolactone. Biotechnol. Bioeng. 2015, 112, 1354-1364.

(28) Ben-David, M.; Elias, M.; Filippi, J.-J.; Duñach, E.; Silman, I.; Sussman, J. L.; Tawfik, D. S. Catalytic Versatility and Backups in Enzyme Active Sites: The Case of Serum Paraoxonase 1. J. Mol. Biol. 2012, 418, 181-196.

(29) Binda, C.; Robinson, R. M.; Martin del Campo, J. S.; Keul, N. D.; Rodriguez, P. J.; Robinson, H. H.; Mattevi, A.; Sobrado, P. An Unprecedented NADPH Domain Conformation in Lysine Monooxygenase $\mathrm{NbtG}$ Provides Insights into Uncoupling of Oxygen Consumption from Substrate Hydroxylation. J. Biol. Chem. 2015, 290, 12676-12688.

(30) Lennon, B. W.; Williams, C. H.; Ludwig, M. L. Twists in Catalysis: Alternating Conformations of Escherichia Coli Thioredoxin Reductase. Science 2000, 289, 1190-1194.

(31) Yachnin, B. J.; Lau, P. C. K.; Berghuis, A. M. The Role of Conformational Flexibility in Baeyer-Villiger Monooxygenase Catalysis and Structure. Biochim. Biophys. Acta, Proteins Proteomics 2016, 1864, 1641-1648.

(32) Romero, E.; Castellanos, J. R.; Mattevi, A.; Fraaije, M. W. Characterization and Crystal Structure of a Robust Cyclohexanone Monooxygenase. Angew. Chem., Int. Ed. 2016, 55, 15852-15855.

(33) Balke, K.; Beier, A.; Bornscheuer, U. T. Hot Spots for the Protein Engineering of Baeyer-Villiger Monooxygenases. Biotechnol. Adv. 2018, 36, 247-263.

(34) Cunningham, B.; Wells, J. High-Resolution Epitope Mapping of hGH-Receptor Interactions by Alanine-Scanning Mutagenesis. Science 1989, 244, 1081-1085.

(35) Bershtein, S.; Segal, M.; Bekerman, R.; Tokuriki, N.; Tawfik, D. S. Robustness-Epistasis Link Shapes the Fitness Landscape of a Randomly Drifting Protein. Nature 2006, 444, 929.

(36) Polyak, I.; Reetz, M. T.; Thiel, W. Quantum Mechanical/ Molecular Mechanical Study on the Mechanism of the Enzymatic Baeyer-Villiger Reaction. J. Am. Chem. Soc. 2012, 134, 2732-2741.

(37) Kamerbeek, N. M.; Fraaije, M. W.; Janssen, D. B. Identifying Determinants of NADPH Specificity in Baeyer-Villiger Monooxygenases. Eur. J. Biochem. 2004, 271, 2107-16.

(38) Beadle, B. M.; Shoichet, B. K. Structural Bases of StabilityFunction Tradeoffs in Enzymes. J. Mol. Biol. 2002, 321, 285-296.

(39) Dudek, H. M.; de Gonzalo, G.; Torres Pazmino, D. E.; Stepniak, P.; Wyrwicz, L. S.; Rychlewski, L.; Fraaije, M. W. Mapping the Substrate Binding Site of Phenylacetone Monooxygenase from Thermobifida Fusca by Mutational Analysis. Appl. Environ. Microbiol. 2011, 77, 5730-5738.

(40) Forneris, F.; Orru, R.; Bonivento, D.; Chiarelli, L. R.; Mattevi, A. Thermofad, a Thermofluor-Adapted Flavin Ad Hoc Detection System for Protein Folding and Ligand Binding. FEBS J. 2009, 276, 2833-2840.

(41) Riebel, A.; Dudek, H. M.; de Gonzalo, G.; Stepniak, P.; Rychlewski, L.; Fraaije, M. W. Expanding the Set of Rhodococcal Baeyer-Villiger Monooxygenases by High-Throughput Cloning, Expression and Substrate Screening. Appl. Microbiol. Biotechnol. 2012, 95, 1479-1489.

(42) Černuchová, P.; Mihovilovic, M. D. Microbial Baeyer-Villiger Oxidation of Terpenones by Recombinant Whole-Cell BiocatalystsFormation of Enantiocomplementary Regioisomeric Lactones. Org. Biomol. Chem. 2007, 5, 1715-1719.

(43) Lowe, J. R.; Tolman, W. B.; Hillmyer, M. A. Oxidized Dihydrocarvone as a Renewable Multifunctional Monomer for the Synthesis of Shape Memory Polyesters. Biomacromolecules 2009, 10, 2003-2008. 
(44) Balke, K.; Baumgen, M.; Bornscheuer, U. T. Controlling the Regioselectivity of Baeyer-Villiger Monooxygenases by Mutation of Active-Site Residues. ChemBioChem 2017, 18, 1627-1638.

(45) Balke, K.; Schmidt, S.; Genz, M.; Bornscheuer, U. T. Switching the Regioselectivity of a Cyclohexanone Monooxygenase toward (+)-Trans-Dihydrocarvone by Rational Protein Design. ACS Chem. Biol. 2016, 11, 38-43.

(46) Messiha, H. L.; Ahmed, S. T.; Karuppiah, V.; Suardíaz, R.; Ascue Avalos, G. A.; Fey, N.; Yeates, S.; Toogood, H. S.; Mulholland, A. J.; Scrutton, N. S. Biocatalytic Routes to Lactone Monomers for Polymer Production. Biochemistry 2018, 57, 1997-2008.

(47) Li, G.; Garcia-Borràs, M.; Fürst, M. J. L. J.; Ilie, A.; Fraaije, M. W.; Houk, K. N.; Reetz, M. T. Overriding Traditional Electronic Effects in Biocatalytic Baeyer-Villiger Reactions by Directed Evolution. J. Am. Chem. Soc. 2018, 140, 10464-10472.

(48) Fürst, M. J. L. J.; Savino, S.; Dudek, H. M.; Gomez Castellanos, J. R.; Gutierrez de Souza, C.; Rovida, S.; Fraaije, M. W.; Mattevi, A. Polycyclic Ketone Monooxygenase from the Thermophilic Fungus Thermothelomyces Thermophila: A Structurally Distinct Biocatalyst for Bulky Substrates. J. Am. Chem. Soc. 2017, 139, 627-630.

(49) Mitchell, L. A.; Cai, Y.; Taylor, M.; Noronha, A. M.; Chuang, J.; Dai, L.; Boeke, J. D. Multichange Isothermal Mutagenesis: A New Strategy for Multiple Site-Directed Mutations in Plasmid DNA. ACS Synth. Biol. 2013, 2, 473-477.

(50) Sykora, J.; Brezovsky, J.; Koudelakova, T.; Lahoda, M.; Fortova, A.; Chernovets, T.; Chaloupkova, R.; Stepankova, V.; Prokop, Z.; Smatanova, I. K.; Hof, M.; Damborsky, J. Dynamics and Hydration Explain Failed Functional Transformation in Dehalogenase Design. Nat. Chem. Biol. 2014, 10, 428.

(51) Cashman, J. R. Some Distinctions between Flavin-Containing and Cytochrome P450 Monooxygenases. Biochem. Biophys. Res. Commun. 2005, 338, 599-604.

(52) Franceschini, S.; Fedkenheuer, M.; Vogelaar, N. J.; Robinson, H. H.; Sobrado, P.; Mattevi, A. Structural Insight into the Mechanism of Oxygen Activation and Substrate Selectivity of Flavin-Dependent N-Hydroxylating Monooxygenases. Biochemistry 2012, 51, 70437045.

(53) McLeod, M. P.; Warren, R. L.; Hsiao, W. W.; Araki, N.; Myhre, M.; Fernandes, C.; Miyazawa, D.; Wong, W.; Lillquist, A. L.; Wang, D.; et al. The Complete Genome of Rhodococcus Sp. Rha1 Provides Insights into a Catabolic Powerhouse. Proc. Natl. Acad. Sci. U. S. A. 2006, 103, 15582-15587.

(54) Bar-Rogovsky, H.; Hugenmatter, A.; Tawfik, D. S. The Evolutionary Origins of Detoxifying Enzymes: The Mammalian Serum Paraoxonases (Pons) Relate to Bacterial Homoserine Lactonases. J. Biol. Chem. 2013, 288, 23914-27.

(55) Khersonsky, O.; Tawfik, D. S. Structure-Reactivity Studies of Serum Paraoxonase Pon 1 Suggest That Its Native Activity Is Lactonase. Biochemistry 2005, 44, 6371-6382.

(56) Ziegler, D. M. Flavin-Containing Monooxygenases: Enzymes Adapted for Multisubstrate Specificity. Trends Pharmacol. Sci. 1990, $11,321-324$.

(57) Girvan, H. M.; Munro, A. W. Applications of Microbial Cytochrome P450 Enzymes in Biotechnology and Synthetic Biology. Curr. Opin. Chem. Biol. 2016, 31, 136-145. 